Pacific Journal of Mathematics

FIXED POINT THEOREMS FOR ACYCLIC AND DENDRITIC

AND RAYMOND EARL SMithson 


\title{
FIXED POINT THEOREMS FOR ACYCLIC AND DENDRITIC SPACES
}

\author{
T. B. Muenzenberger and R. E. SMithson
}

\begin{abstract}
A mod is a partially ordered set $(X, \leqq)$ such that: (i) $x \wedge y$ exists for all $x, y \in X$. (ii) $L(x)$ is totally ordered for all $x \in X$. (iii) $(X, \leqq)$ is conditionally complete and order dense. Fixed point theorems for certain functions on totally ordered mods are extended to multifunctions on arbitrary mods by using weak continuity conditions. Characterizations of continuity are also given for certain functions on mods. Mods are shown to be algebraic models for acyclic and dendritic spaces.
\end{abstract}

1. Introduction. A number of papers giving fixed point theorems for decreasing or increasing functions on partially ordered sets have appeared in the last twenty years. For example there is Tarski's classic result for increasing functions on a lattice [15] and Davis' subsequent proof of the converse [3]. Abian and Brown extended Tarski's theorem to more general partially ordered sets [2], and in [11] and [13] Smithson further extended the results of [2], [3], and [15]. Ward used the fixed point property for increasing functions to characterize compactness of the interval topology in semi-lattices [16]. (See also [6] in this regard.) Abian gave a sufficient condition for decreasing functions on totally ordered sets to have fixed points [1], and then Metcalf and Payne extended Abian's result to include functions which were neither decreasing nor increasing [4].

It will be shown that certain highly technical conditions used by Abian, Metcalf and Payne are forms of continuity which parallel assumptions used by Muenzenberger and Smithson in another context, where the underlying algebraic structure common to trees was isolated in the concept of a semitree and fixed point theorems were proved for multifunctions satisfying weak continuity conditions on semitrees [5]. Further the fixed point theorem of Abian, Metcalf, and Payne will be extended to include multifunctions on non totally ordered sets.

In the following $(X, \leqq)$ will denote a nonempty partially ordered set $X$ with partial order $\leqq$. A subset $A$ of $X$ is a toset just in case $A$ is totally ordered. Infima and suprema are defined in the usual way, and $x<y$ means $x \leqq y$ and $x \neq y$. For $x \in X$ and $A \subset X$, define $L(x)=\{y: y \leqq x\}, M(x)=\{y: x \leqq y\}$, and $M(A)=\bigcup\{M(x): x \in A\}$. Also $x \wedge y=\inf \{x, y\}$ when the latter exists. A function $f: X \rightarrow X$ is decreasing (increasing) if and only if for all $x, y \in X, x \leqq y$ implies 
$f(y) \leqq f(x)(f(x) \leqq f(y))$

Definition. A partially ordered set $(X, \leqq)$ is a mod if and only if the following hold:

(i) For all $x, y \in X, x \wedge y$ exists.

(ii) For all $x \in X, L(x)$ is a toset.

(iii) Each nonempty subset of $X$ which is bounded above (below) has a supremum (infimum) in $X$.

(iv) If $x<y$, then there is a $z \in X$ such that $x<z<y$.

The word mod derives from the terms triod, $m-o d$, and so forth. We shall frequently refer to the $\bmod X$ when we mean the $\bmod (X, \leqq)$.

In the language of [5] and [7], a mod is just a semitree perhaps with some endpoints erased.

For $x, y$ in a $\bmod X$, define

$$
[x, y]= \begin{cases}L(y) \cap M(x) & \text { if } x \leqq y \\ {[x \wedge y, x] \cup[x \wedge y, y]} & \text { if } x \text { and } y \text { are not comparable }\end{cases}
$$

The set $[x, y]$ is called the chain with endpoints $x$ and $y$. A set $A \subset X$ is chainable if and only if $x, y \in A$ implies $[x, y] \subset A$. For $A, B \subset X$, define

$$
[A, B]=\bigcup\{[x, y]: x \in A \text { and } y \in B\} .
$$

It turns out that the family of chains completely determines the structure of a mod.

Before continuing the development of the fixed point theorems we shall show that two important and large classes of spaces admit natural mod structures. Thus the following fixed point theorems apply to these spaces.

Definition. A Hausdorff space $X$ is acyclic if and only if each two distinct points $x, y$ in $X$ are the endpoints of a unique arc $[x, y]$ in $X$. (In this context arcs need not be separable.)

If $X$ is an acyclic, space then we define a partial order $\leqq$ on $X$ as follows: Fix an element $e \in X$. Then, for $x, y \in X, x \leqq y$ if and only if $x \in[e, y]$. The proof that $\leqq$ is a partial order is straightforward and is omitted.

THeOREM 1.1. If $X$ is an acyclic space, then $(X, \leqq)$ satisfies conditions (i) through (iv). That is, an acyclic space admits the structure of a mod.

Proof. Since the partial order $\leqq$ has a least element $e$, condition 
(iii) implies (i). Next note that since $X$ is acyclic, $L(x)=[e, x]$ for each $x$ and thus is totally ordered. Hence, (ii) holds. Also since $L(x)$ is the arc $[e, x]$, the partial order is order dense. Moreover, suppose that $A$ is a nonempty subset of $X$ which is bounded above. Then $A \subset L(x)=[e, x]$ for some $x \in X$. Then $a_{0}=\sup A$ exists in $L(x)$. If $b$ is another upper bound for $A$, then $A \subset L(b) \cap L(x)$ which is a compact subset of $L(x)$ and hence, $a_{0} \in L(b) \cap L(x)$. Thus $a_{0}$ is the supremum of $A$ in $X$. Finally let $A$ be any nonempty subset of $X$ and let $B=\bigcap\{L(a): a \in A\}$. Then $B \subset L(a)$ for each $a \in A$ and $b_{0}=\sup B \in L(\alpha)$ for each $a \in A$. Further, if $x \in L(\alpha)$ for each $a \in A$, then $x \in B$ and hence, $b_{0}=\inf A$. Thus (iii) holds.

Definition. A connected Hausdorff space is called dendritic if and only if any two distinct points can be separated by the omission of a third point.

A dendritic space $X$ is given a partial order as follows: Fix an element $e \in X$, then $x \leqq y$ if and only if $x=e, x=y$ or $x$ separates $e$ and $y$. Dendritic spaces and their order structure have been studied extensively by Ward in [17]. In [17] Ward establishes much of what is needed to show that a dendritic space with the above partial order is a mod. Unfortunately, there is a gap in the proof of one of the key propositions in [17] (Lemma 8.2). In effect what is needed to complete the proof of Lemma 8.2 in [17] is: If $x, y$ are any two elements of $X$, then $\inf \{x, y\}$ exists in $X$. The proof of this latter statement is fairly involved and is given by Muenzenberger and Smithson in [8]. Then, given this, from Lemma 8.2 of [17] we get that each nonempty set which is bounded above has a supremum in $X$ and this implies that each nonempty set has an infimum in $X$. That $L(x)$ is a chain follows from Theorem 1 of [17] and the fact that $\leqq$ is order dense is given by Theorem 3 of [17]. Thus we have:

Theorem 1.2. A dendritic space admits the structure of a mod.

2. Natural topologies on mods. Let $X$ be a mod throughout this section. The lower (upper) interval topology $\mathscr{T}_{L}\left(\mathscr{T}_{M}\right)$ is that with subbasis for the closed sets consisting of all $L(x)(M(x))$ for $x \in$ $X$. The interval topology $\mathscr{T}_{I}$ is generated by $\mathscr{T}_{L} \cup \mathscr{T}_{M}$. A set $C \subset X$ is closed under infima (suprema) precisely when $\inf A \in$ $C(\sup A \in C)$ for every nonempty toset $A \subset D$ which is bounded below (above). The inf (sup) topology $\mathscr{F}(\mathscr{S})$ is that whose closed sets are exactly those which are closed under infima (suprema). The chain topology $\mathscr{C}$ is $\mathscr{J} \cap \mathscr{S}$. Equivalently $C \subset X$ is $\mathscr{C}$ closed if and only if $\inf A, \sup A \in C$ for every bounded nonempty set $A \subset C$. The 
topology $\mathscr{C}$ arose naturally in studying semitrees [5], [7]. In general $\mathscr{T}_{I} \subset \mathscr{C}$, and $\mathscr{T}_{I}=\mathscr{C}$ in a totally ordered mod.

DEFINITION. A function $f: X \rightarrow X$ is nonoscillatory from above if and only if for each nonmaximal $x$ and each maximal toset $A \subset$ $M(x)-\{x\}$,

$$
\cap\{f([x, u]): u \in A\}=\{f\{x)\} .
$$

The function $f$ is nonscillatory from below if and only if for each nonminimal $x$,

$$
\cap\{f([u, x]): u<x\}=\{f(x)\} .
$$

This definition could be made for multifunctions, and the following results would remain valid. See [4] for information and intuition regarding these two concepts of Metcalf and Paynh. We limit ourselves to proving that they are weak continuity conditions.

\section{THEOREM 2.1. Let $f: X \rightarrow X$ where $X$ is a mod. Then}

(i) $f$ is nonoscillatory from above if and only if $f^{-1}(x)$ is $\mathcal{F}$ closed for all $x \in X$.

(ii) $f$ is nonoscillatory from below if and only if $f^{-1}(x)$ is $\mathscr{S}$ closed for all $x \in X$.

Proof. Suppose that $f$ is nonoscillatory from below. Let $A$ be a nonempty subset of $f^{-}(x)$ which is bounded above and let $a=\sup A$. For any $u<a$, there is $v \in f^{-1}(x)$ such that $u<v<a$. Then $x \in \cap$ $\{f([u, a]): u<a\}$. So $x=f(a)$ since $f$ is nonoscillatory from below, and $f^{-1}(x)$ is $\mathscr{S}$ closed. Suppose conversely that $f^{-1}(x)$ is $\mathscr{S}$ closed for all $x \in X$ and let $v \in \cap\{f([u, x]): u<x\}$. Then for $u<x$ there is a set $B \subset[u, x] \cap f^{-1}(v)$ such that $x=\sup B$. But $f^{-1}(v) \mathscr{S}$ closed implies that $v=f(x)$, which completes the proof of (ii). The proof of (i) is similar to the above proof and so is omitted.

Corollary 2.2. Let $X$ be a mod. A function $f: X \rightarrow X$ is nonoscillatory from above and below if and only if $f^{-1}(x)$ is $\mathscr{C}$ closed for all $x \in X$.

Corollary 2.3. Let $X$ be a mod and $f: X \rightarrow X$. If $f^{-1}(x)$ is $\mathscr{T}_{I}, \mathscr{T}_{L}$, or $\mathscr{T}_{M}$ closed for each $x \in X$, then $f$ is nonoscillatory from above and below.

Corollary 2.4. Let $X$ be a totally ordered mod. A function $f: X \rightarrow X$ is nonoscillatory from above and below if and only if $f^{-1}(x)$ is $\mathscr{T}_{I}$ closed for all $x \in X$. 
It is easy to see that the converse of Corollary 2.3 is false, and so it is necessary that $X$ be totally ordered in Corollary 2.4. In the next section we shall need the following lemma which was proved in less generality in [5].

Lemma 2.5. Let $X$ be a mod and let $C \subset X$. If $C$ is bounded below, chainable, $\mathscr{F}$ closed, and nonempty, then inf $C \in C$.

Proof. Let $c_{0}=\inf C$ and let $c \in C$. Then $A=C \cap\left[c_{0}, c\right]$ is a nonempty toset in $C$ and hence $a=\inf A \in C$. If $c_{0}=a$, then we are done. Thus suppose that $c_{0}<a$. Then there exists $x \in C$ such that $a \nsupseteq x$. But $c_{0} \leqq a \wedge x<a$ and $a \wedge x \in C$ since $C$ is chainable. This contradicts $a=\inf A$ and completes the proof.

3. A fixed point theorem. In this section we prove an extension of the main theorems in [1], [4], [5], and [12]. We do not assume that $X$ is totally ordered in the main theorem, but we do assume the existence of maximal elements. An example will show this is necessary. The setting in our theorem is essentially a semitree perhaps with the least element erased.

THEOREM 3.1. Let $X$ be a mod in which every nonempty toset has a supremum. Suppose that $F: X \rightarrow X$ satisfies:

(i) For all $x \in X, F(x)$ is chainable and $\mathscr{C}$ closed.

(ii) Every nondegenerate chain contains a nonendpoint $x$ such that $F^{-1}(x)$ is either $\mathscr{I}$ closed ( $\mathscr{S}$ closed) or chainable.

(iii) If $x \leqq y$ and $F(y) \cap M(F(x))=\varnothing$, then $[F(x), F(y)] \subset$ $F([x, y])$.

(iv) There exists $x_{0} \in X$ such that $F\left(x_{0}\right) \cap M\left(x_{0}\right) \neq \varnothing$. Then $F$ has a fixed point.

Proof. First assume that every nondegenerate chain contains a nonendpoint $x$ such that $F^{-1}(x)$ is either $\mathscr{S}$ closed or chainable. Let

$$
S=\{x \in X: F(x) \cap M(x) \neq \varnothing\} .
$$

Let $A$ be a maximal toset in $S$ and set $a=\sup A$. Note that if $F(x) \cap M(x) \neq \varnothing$ and $x \notin F(x)$, then $F(x) \subset M(x)$. So we have two cases to consider.

I. Suppose that $F(a) \subset M(a)$. Let $i=\inf F(a)$. By Lemma 2.5, $i \in F(a)$. Suppose $a<i$ and pick $x$ so that $a<x<i$. For any $y \in F(x), i \wedge y<x<i$. So $x \in[i, y] \subset[F(x), F(a)] \subset F([a, x])$. Thus $x \in F(z)$ for some $z$ satisfying $a \leqq z \leqq x$. Now $z=a$ is impossible, 
$z=x$ means that $x$ is a fixed point, and $a<z<x$ contradicts $a=$ sup $A$ since $z \in S$. Therefore in any event we have a fixed point.

II. Suppose that $F(a) \cap M(a)=\varnothing$. Since $F(a)$ is $\mathscr{C}$ closed, there exists $b<a$ such that $F(a) \cap[b, a]=\varnothing$, and since $F(a)$ is chainable, we may in fact assume that $F(a) \cap M([b, a])=\varnothing$. By (ii) we may further assume that $F^{-1}(b)$ is either $\mathscr{S}$ closed or chainable. For any $x$ satisfying $b<x<a$ and $F(x) \cap M(x) \neq \varnothing$, either $x$ is a fixed point or else $b \in[F(x), F(a)] \subset F([x, a])$. In the later event, there exists $y$ such that $x<y<a$ and $b \in F(y)$. Applying the argument repeatedly, we locate a fixed point of $F$ or else we construct a toset $B \subset F^{-1}(b) \cap[b, a]$ such that $\alpha=\sup B$. If $F^{-1}(b)$ is $\mathscr{S}$ closed, then $a \in F^{-1}(b)$ which is a contradiction. If $F^{-1}(b)$ is chainable, then there exists a point $c$ such that $b<c<a$ and $[c, a]-\{a\} \subset F^{-1}(b)$. Choose $x$ so that $c<x<a$ and $F(x) \cap M(x) \neq \varnothing$. But $b \in F(x)$ implies that $x \in F(x)$ since $F(x)$ is chainable. So we have a fixed point.

The case where every nondegenerate chain contains a nonendpoint $x$ such that $F^{-1}(x)$ is either $\mathscr{J}$ closed or chainable is handled by considering the set

$$
I=\left\{x \in X: x_{0} \leqq x \text { and } x_{0} \leqq z \leqq x \text { implies } F(z) \cap M(z) \neq \varnothing\right\} .
$$

The argument here is dual to the above as it amounts to turning $X$ upside down. It is similar to one in [5] and is therefore omitted.

Question. Can (ii) replaced by the following?

(ii) $^{\prime}$ Every nondegenerate chain contains a nonendpoint $x$ such that $F^{-1}(x)$ is either $\mathscr{I}$ closed, $\mathscr{S}$ closed, or chainable.

The reader may easily construct simple examples of functions on the unit interval that satisfy (ii)' and all conditions in Theorem 3.1 except (ii). It is also seen that no hypothesis on $F$ in Theorem 3.1 can be dropped. Moreover the hypotheses on $X$ are essential.

ExAmPle. Let $X$ be the triod

$$
\{(x, 0):-1 \leqq x<1\} \cup\{(0, y): 0 \leqq y \leqq 1\}
$$

in the plane and let $\leqq$ be the cut point partial order on $X$ with least element $(-1,0)$. We let $f: X \rightarrow X$ be any fixed point free function that pushes the two closed arms of the triod over into the half open arm and slides the later toward the missing maximal element. Specifically let 


$$
f((x, y))=\left(\frac{1}{2}+\frac{1}{2}(x-y), 0\right) \text { for } \quad(x, y) \in X
$$

Observe that $f$ satisfies all four conditions in Theorem 3.1. The problem is that $X$ contains a nonempty toset with no supremum. Moreover, there exists $b \in X$ such that $f(b) \leqq b$. So it is necessary that $X$ be totally ordered in the following corollary due to Metcalf and Payne [4].

Corollary 3.2. Let $X$ be a totally ordered mod. Suppose that $f: X \rightarrow X$ is a function satisfying:

(i) If $x \leqq y$ and if $f(y) \leqq f(x)$, then $[f(y), f(x)] \subset f([x, y])$.

(ii) The function $f$ is either nonoscillatory from above or from below.

(iii) There exist $a, b \in X$ such that $a \leqq b, a \leqq f(a)$, and $f(b) \leqq b$. Then $f$ has a fixed point.

Proof. Using Theorem 2.1, we have all the necessary conditions on $f$ to apply Theorem 3.1, and the requirement that every nonempty toset have a supremum is replaced by the existence of $b$. A suitable modification of the above proof finishes the argument here.

4. Decreasing functions. In this section we shall prove some results which will be required in $\S 5$.

Definition. Let $X$ be a mod (topological space). A multifunction $F: X \rightarrow X$ is chainable (connected) if and only if $F(C)$ is chainable (connected) for each chainable (connected) set $C \subset X$. Further $F$ is point chainable (closed) if and only. if $F(x)$ is chainable (closed) for each $x \in X$.

The first lemma is a folk theorem in topology [9], and it does not extend to multifunctions.

Lemma 4.1. Let $f: X \rightarrow Y$ where $X, Y$ are Hausdorff spaces. If $f$ is connected and $f^{-1}(y)$ is connected for all $y \in Y$, then $f^{-1}(y)$ is closed for all $y \in Y$.

The second lemma does extend to multifunctions on more general spaces [10].

LemMA 4.2. Let $f: X \rightarrow X$ where $(X, \mathscr{C})$ is a tolly ordered mod. Then $f$ is continuous if and only if $f$ is connected and $f^{-1}(x)$ is closed for all $x \in X$. 
Corollary 4.3. Let $f: X \rightarrow X$ where $(X, \mathscr{C})$ is a totally ordered mod. If $f$ is connected and decreasing, then $f^{-1}(x)$ is connected and closed for each $x \in X$. Hence $f$ is continuous.

Proof. Let $a, b \in f^{-1}(x)$ where $a \leqq b$ and let $c \in[a, b]$. Then $x=f(b) \leqq f(c) \leqq f(\alpha)=x$. Hence $f(c)=x$. So $f^{-1}(x)$ is chainable and therefore connected. (In fact chainability and connectedness coincide in a totally ordered $\bmod (X, \mathscr{C})$.) Apply Lemmas 4.1 and 4.2 .

We next demonstrate that the following condition introduced by Abian [1] is a form of continuity.

Abian's condition. Let $f: X \rightarrow X$ where $X$ is a mod. If $A \subset X$ is a toset, then

$$
f(\inf A)=\sup f(A) \text { and } f(\sup A)=\inf f(A)
$$

whenever both sides of the equalities exist.

THEOREM 4.4. Let $f: X \rightarrow X$ be decreasing where $(X, \mathscr{C})$ is a mod. Then $f$ is continuous if and only if $f$ satisfies Abian's condition.

Proof. Suppose that $f$ is continuous. Let $A \subset X$ be a toset and let $a=\sup A$. Then $f(a)$ is a lower bound for $f(A)$ and thus $f(a) \leqq \inf f(A)$. Suppose that $f(\alpha)<\inf f(A)$. Pick $p$ so that $f(a)<$ $p<\inf f(A)$. Then $M(p)$ is a closed set which contains $f(A)$ and does not contain $f(a)$. This is a contradiction since $a \in A^{*}$ implies $f(a) \in f\left(A^{*}\right) \subset f(A)^{*}$. Hence $f(\sup A)=\inf f(A)$. An analogous argument shows the other equality. Suppose conversely that $f$ satisfies Abian's condition. Let $C \subset X$ be closed and let $A \subset f^{-1}(C)$ be bounded and nonempty. Then $f(A)$ is bounded, $\sup f(A)=f(\inf A)$, and $\inf f(A)=f(\sup A)$. Since $f(A) \subset C$ which is closed, inf $f(A)$ and $\sup f(A)$ are in $C$. Thus $\inf A$ and $\sup A$ are in $f^{-1}(C)$ completing the proof.

A similar argument establishes the following.

THEOREM 4.5. Let $f: X \rightarrow X$ be increasing where $(X, \mathscr{C})$ is a mod. Then $f$ is continuous if and only if for every toset $A \subset X$, $f(\inf \mathrm{A})=\inf f(A)$ and $f(\sup A)=\sup f(A)$ whenever both sides of the equalities exist.

We now generalize the concept of decreasing function. Observe that for functions the following definition reduces to the prior one. 
Definition. Let $X$ be a mod. Then $F: X \rightarrow X$ is decreasing if and only if $x \leqq y$ implies

1. For all $z \in F(x), L(z) \cap F(y) \neq \varnothing$.

2. For all $z \in F(y), M(z) \cap F(x) \neq \varnothing$.

Lemma 4.6. Let $F: X \rightarrow X$ be decreasing where $X$ is a nonempty mod. Then there exist $a, b \in X$ such that $a \leqq b \in F(a)$ and $F(a) \cap$ $M(a) \neq \varnothing \neq F(b) \cap L(b)$.

Proof. Let $x \in X$. If $F(x) \cap M(x) \neq \varnothing$, then take $a=x$ and $b \in F(a) \cap M(a)$. If $F(x) \cap M(x)=\varnothing$, then take $y \in F(x)$ and set $a=$ $x \wedge y$. By condition 2, there is $b \in M(y) \cap F(a)$. In either case $a$ and $b$ have the desired properties.

To indicate some of the flavor of other results obtainable for decreasing functions on mods, we state but do not prove two theorems that perhaps reflect their progenitors stated above.

Theorem 4.7. Let $f: X \rightarrow X$ where $(X, \mathscr{C})$ is a mod. If $f$ is chainable and $f^{-1}(x)$ is chainable for all $x \in X$, then $f^{-1}(x)$ is closed for all $x \in X$.

THEOREM 4.8. Let $F: X \rightarrow X$ where $X$ is a totally ordered mod. If $F$ is decreasing and point chainable, then $F^{-1}(x)$ is chainable for each $x \in X$.

The last theorem does not extend to arbitrary mods.

5. Fixed points for decreasing multifunctions, In this section we derive Abian's result [1] from Theorem 3.1 and then prove some generalizations that are independent of Theorem 3.1. The first lemma is similar to one in [14] and follows from Corollary 3.2.

Lemma 5.1. Let $f: X \rightarrow X$ be continuous where $(Y, \mathscr{C})$ is a totally ordered mod. If there exist $a, b \in X$ such that $a \leqq b, a \leqq f(a)$, and $f(b) \leqq b$, then $f$ has a fixed point.

Lemma 5.2. Let $f: X \rightarrow X$ where $(X, \mathscr{C})$ is a totally ordered mod. If $f$ is connected and decreasing, then $f$ has a fixed point.

Proof. Let $x \in X$. If $x \leqq f(x)$, then take $a=x$ and $b=f(x)$. If $f(x) \leqq x$, then set $a=f(x)$ and $b=x$. In either case apply Corollary 4.3 and Lemma 5.1 .

Abian's theorem [1] then follows from Theorem 4.4 and Lemma 5.2 . 
CoRollary 5.3. Let $f: X \rightarrow X$ where $X$ is a totally ordered mod. If $f$ is decreasing and satisfies Abian's condition, then $f$ has a fixed point.

We now generalize Abian's theorem to decreasing multifunctions on arbitrary mods.

Theorem 5.4. Let $F: X \rightarrow X$ where $(X, \mathscr{C})$ is a mod. If $F$ is chainable, decreasing, and point closed, then $F$ has a fixed point.

Proof. From Lemma 4.6 there exist $a, b \in X$ such that $a \leqq b €$ $F(a)$ and $F(a) \cap M(a) \neq \varnothing \neq F(b) \cap L(b)$. Set

$$
S=\{x \in X: a \leqq x<b \text { and } F(x) \cap M(x) \neq \varnothing\}
$$

and let $c=\sup S$. Again we have two cases to consider.

I. Suppose that $F(c) \subset M(c)$ and let $i=\inf F(c)$. By Lemma 2.5, $i \in F(c)$. Suppose $c<i$ and pick $x$ so that $c<x<i$. But the chainable set $F([c, x])$ meets $M(x)$ and its complement. So $x \in F([c, x])$, and this case is completed as in the proof of Theorem 3.1.

II. Suppose that $F(c) \cap M(c)=\varnothing$. Since $F(c)$ is $\mathscr{C}$ closed, there exists $x_{0}<c$ such that $F(c) \cap\left[x_{0}, c\right]=\varnothing$, and since $F(c)$ is chainable, we may assume that $F(c) \cap M\left(\left[x_{0}, c\right]\right)=\varnothing$. Let $u$ satisfy $x_{0}<u<c$ and $F(u) \cap M(u) \neq \varnothing$. Now the chainable set $F([u, c])$ meets $M(u)$ and its complement. So there exists $x$ such that $u \leqq x<c$ and $u \in$ $F(x)$. Pick $v$ to satisfy $x<v<c$ and $F(v) \cap M(v) \neq \varnothing$. But $u \in F(x)$ implies $F(v) \cap L(u) \neq \varnothing$ since $F$ is decreasing. Thus $F(v)$ meets $M(v)$ and $L(v)$. So $v$ is a fixed point.

COROLLARY 5.5. Every chainable dereasing function on a mod into itself has a fixed point.

By slight modifications of the hypotheses, a plethora of hybrids of Theorem 3.1 and 5.4 can be obtained. We indicate but two examples of these.

TheOREm 5.6 Let $(X, \mathscr{C})$ be a mod and suppose that $F: X \rightarrow X$ satisfies:

(i) $F$ is point chainable and point closed.

(ii) $F$ is decreasing.

(iii) If $x \leqq y$ and $F(y) \cap M(F(x))=\varnothing$, then $F([x, y])$ is chainable. Then $F$ has a fixed point. 
THEOREM 5.7. Let $(X, \mathscr{C})$ be a mod and suppose that $F: X \rightarrow X$ satisfies:

(i) $F$ is point closed.

(ii) If $x<y$, then $F(y) \cap M(F(x))=\varnothing$.

(iii) $F$ is chainable.

(iv) There exists $x_{0} \in X$ such that $F\left(x_{0}\right) \cap M\left(x_{0}\right) \neq \varnothing$. Then $F$ has a fixed point.

ExAmple. 1. Let $X=[0,1]$ and define $F: X \rightarrow X$ by $F(x)=1$ if $0 \leqq x<1$ and $F(1)=[0,1)$. Then $F$ is chainable and decreasing but does not have a fixed point. So the point closed hypothesis is necessary in the above theorems.

2. If $X=[0,1]$ and $F$ is defined by $F(1)=[0,1 / 2]$ and $F(x)=$ $[1 / 2,1]$ for $0 \leqq x<1$, then $F$ is chainable, decreasing, and point closd but $F^{-1}(1)$ is not closed. Thus Lemma 4.1, Corollary 4.3, and Theorem 4.7 do not extend to multifunctions. In particular a chainable decreasing point closed multifunction on a totally ordered mod need not be upper semicontinuous, but it must have a fixed point.

\section{REFERENCES}

1. A. Abian, A fixed point theorem for nonincreasing mappings, Boll. Un. Mat. Ital., 2 (1969), 200-201.

2. S. Abian and A. B. Brown, A theorem on partially ordered sets, with applications to fixed point theorem, Canad. J. Math., 13 (1961), 78-82.

3. A. C. Davis, A characterization of complete lattices, Pacific J. Math., 5 (1955), 311-319.

4. F. T. Metcalf and T. H. Payne, On the existence of fixed points in a totally ordered set, Proc. Amer. Math. Soc., 31 (1972), 441-444.

5. T. B. Muenzenberger and R. E. Smithson, Fixed point structures, Trans. Amer. Math. Soc., 184 (1973), 153-173.

6. - Characterizations of compactness of the interval topology in semilattices, Proc. Amer. Math. Soc., 46 (1974), 133-136.

7. - The structure of nested space, Trans. Amer. Math. Soc., 201 (1975), 57-87.

8. - Semilattice structures a note on dendritic spaces, (to appear).

9. W. J. Pervin and N. Levine, Connected mappings of Hausdorff spaces, Proc. Amer. Math. Soc., 9 (1958), 488-496.

10. R. E. Smithson, On criteria for continuity, Nieuw Arch. Wisk., 14 (1966) 89-92.

11. - Fixed points of order preserving multifunctions, Proc. Amer. Math.

Soc., 28 (1971), 304-310.

12. - Fixed point theorems for certain classes of multifunctions, Proc. Amer. Math. Soc., 31 (1972), 595-600.

13. - Fixed points in partially ordered sets, Pacific J. Math., 45 (1973), 363-367.

14. R. E. Smithson and L. E. Ward, Jr., The fixed point property for arcwise connected spaces: a correction, Pacific J. Math., 43 (1972), 511-514.

15. A. Tarski, A lattice-theoretical fixpoint theorem and its applications, Pacific J. Math., 5 (1955), 285-309.

16. L. E. Ward, Jr., Completeness in semilattices, Canad. J. Math., 9 (1957), 578-582. 
17. - Recent Developments in Dendritic Spaces and Related Topics, Studies in Topology (Proc. Conf., University of North Carolina, Charlotte, North Carolina), Academic Press, New York, 1975, 601-647.

Received February 2, 1977. Supported in part by a University of Wyoming Graduate School Summer Fellowship.

Kansas State University

MANHATTAN, KS 66506

UNIVERSITY OF HOUSTON

AND

UNIVERSITY OF WYOMING 


\title{
PACIFIC JOURNAL OF MATHEMATICS
}

\section{EDITORS}

\author{
RICHARD ARENS (Managing Editor) \\ University of California \\ Los Angeles, CA 90024 \\ Charles W. Curtis \\ University of Oregon \\ Eugene, OR 97403 \\ C. C. MOORE \\ University of California \\ Berkeley, CA 94720
}

\section{J. DUGUNDJI}

Department of Mathematics

University of Southern California

Los Angeles, CA 90007

R. FINN and J. MILGRAM

Stanford University

Stanford, CA 94305

\section{ASSOCIATE EDITORS}
E. F. BECKENBACH
B. H. NeumanN
F. WOLF
K. YoSHIDA

\section{SUPPORTING INSTITUTIONS}

UNIVERSITY OF BRITISH COLUMBIA

CALIFORNIA INSTITUTE OF TECHNOLOGY

UNIVERSITY OF CALIFORNIA

MONTANA STATE UNIVERSITY

UNIVERSITY OF NEVADA, RENO

NEW MEXICO STATE UNIVERSITY

OREGON STATE UNIVERSITY

UNIVERSITY OF OREGON

OSAKA UNIVERSITY

\author{
UNIVERSITY OF SOUTHERN CALIFORNIA \\ STANFORD UNIVERSITY \\ UNIVERSITY OF HAWAII \\ UNIVERSITY OF TOKYO \\ UNIVERSITY OF UTAH \\ WASHINGTON STATE UNIVERSITY \\ UNIVERSITY OF WASHINGTON \\ $\stackrel{*}{*} \stackrel{*}{*} \stackrel{*}{*}$ AMERICAN MATHEMATICAL SOCIETY
}

The Supporting Institutions listed above contribute to the cost of publication of this Journal, but they are not owners or publishers and have no responsibility for its content or policies.

Mathematical papers intended for publication in the Pacific Jaurnal of Mathematics should be in typed form or offset-reproduced, (not dittoed), double spaced with large margins. Please do not use built up fractions in the text of your manuscript. You may however, use them in the displayed equations. Underline Greek letters in red, German in green, and script in blue. The first paragraph or two must be capable of being used separately as a synopsis of the entire paper. Items of the bibliography should not be cited there unless absolutely necessary, in which case they must be identified by author and Journal, rather than by item number. Manuscripts, in triplicate, may be sent to any one of the editors. Please classify according to the scheme of Math. Reviews, Index to Vol. 39. All other communications should be addressed to the managing editor, or Elaine Barth, University of California, Los Angeles, California, 90024.

The Pacific Journal of Mathematics expects the author's institution to pay page charges, and reserves the right to delay publication for nonpayment of charges in case of financial emergency.

100 reprints are provided free for each article, only if page charges have been substantially paid. Additional copies may be obtained at cost in multiples of 50 .

The Pacific Journal of Mathematics is issued monthly as of January 1966. Regular subscription rate: $\$ 7200$ a year (6 Vols., 12 issues). Special rate: $\$ 36.00$ a year to individual members of supporting institutions.

Subscriptions, orders for back numbers, and changes of address should be sent to Pacific Journal of Mathematics, 103 Highland Boulevard, Berkeley, California, 94708.

PUBLISHED BY PACIFIC JOURNAL OF MATHEMATICS, A NON-PROFIT CORPORATION

Printed at Kokusai Bunken Insatsusha (International Academic Printing Co., Ltd.). 8-8, 3-chome, Takadanobaba, Shinjuku-ku, Tokyo 160, Japan.

Copyright (C) 1975 by Pacific Journal of Mathematics Manufactured and first issued in Japan 


\section{Pacific Journal of Mathematics}

Vol. 72, No. 2

February, 1977

George E. Andrews, Plane partitions. II. The equivalence of the

Bender-Knuth and MacMahon conjectures ................. 283

Lee Wilson Badger, An Ehrenfeucht game for the multivariable quantifiers

of Malitz and some applications ......................... 293

Wayne C. Bell, A decomposition of additive set functions ............ 305

Bruce Blackadar, Infinite tensor products of $C^{*}$-algebras ............. 313

Arne Brøndsted, The inner aperture of a convex set .............. 335

N. Burgoyne, Finite groups with Chevalley-type components........... 341

Richard Dowell Byrd, Justin Thomas Lloyd and Roberto A. Mena, On the retractability of some one-relator groups .....................

Paul Robert Chernoff, Schrödinger and Dirac operators with singular potentials and hyperbolic equations .................... 361

John J. F. Fournier, Sharpness in Young's inequality for convolution ....... 383

Stanley Phillip Franklin and Barbara V. Smith Thomas, On the metrizability

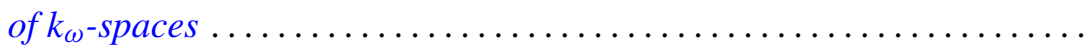

David Andrew Gay, Andrew McDaniel and William Yslas Vélez, Partially normal radical extensions of the rationals .................... 403

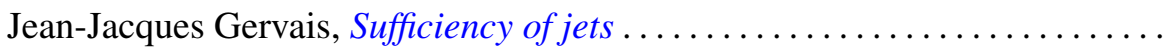

Kenneth R. Goodearl, Completions of regular rings. II . .

Sarah J. Gottlieb, Algebraic automorphisms of algebraic groups with stable maximal tori

Donald Gordon James, Invariant submodules of unimodular Hermitian forms.....

J. Kyle, $W_{\delta}(T)$ is convex.

Ernest A. Michael and Mary Ellen Rudin, A note on Eberlein compacts ...

Ernest A. Michael and Mary Ellen Rudin, Another note on Eberlein compacts ....

Thomas Bourque Muenzenberger and Raymond Earl Smithson, Fixed point theorems for acyclic and dendritic spaces.

Budh Singh Nashier and A. R. Rajwade, Determination of a unique solution of the quadratic partition for primes $p \equiv 1(\bmod 7)$.

Frederick J. Scott, New partial asymptotic stability results for nonlinear ordinary differential equations ....................

Frank Servedio, Affine open orbits, reductive isotropy groups, and dominant gradient morphisms; a theorem of Mikio Sato..........

D. Suryanarayana, On the distribution of some generalized square-full integers.................................. 